\title{
System Frequency Support through Multi-terminal DC (MTDC) Grids
}

\author{
Nilanjan Ray Chaudhuri, Member, IEEE, Rajat Majumder, Member, IEEE, Balarko Chaudhuri, Senior \\ Member, IEEE
}

\begin{abstract}
Control of the converter stations in a multi-terminal DC (MTDC) grid to provide frequency support for the surrounding AC systems is the subject matter of this paper. The standard autonomous power sharing control loop for each converter is modified with a frequency droop control loop. The objective is to minimize the deviation from nominal $\mathrm{AC}$ system frequency and share the burden of frequency support among the converter stations of the MTDC grid. The effectiveness of the frequency support is demonstrated through nonlinear simulation of a test system consisting of three isolated $\mathrm{AC}$ systems interconnected through an MTDC grid with four converter stations. An averaged model of the MTDC grids is developed to carry out modal analysis of combined multi-machine AC-MTDC systems. Modal analysis is used to characterize and substantiate the time domain behavior in presence of frequency droop control. It is established that appropriate droop control loop for the MTDC grid converters could be effective in reducing the deviation from nominal AC system frequency provided the sensitivity of the system eigen-values to changes in control parameters (e.g. droop coefficients) is accounted for apriori through modal analysis.
\end{abstract}

Index Terms-Multi-terminal DC (MTDC), Autonomous power sharing, Droop, Frequency support, Voltage source converter (VSC), Stability, Modal analysis

\section{INTRODUCTION}

$\mathbf{M}$ ULTI-TERMINAL DC (MTDC) grid is becoming increasingly relevant especially in Europe with a panEuropean supergrid project envisaged to interconnect the offshore wind resources to the onshore grid [1]. Due to certain advantages, the voltage source converter (VSC) technology is preferred over its current source counterpart for such DC grids. However, quite a few technical challenges remain to be solved before MTDC grids are realized in practice. This includes the absence of a commercial DC circuit breaker at high power levels. From a system operator's standpoint, the issues like sudden loss of infeed of the order of gigawatts (e.g. from wind farms), autonomous power sharing and interaction amongst the DC and the surrounding AC systems are primary concerns.

Disturbances in the AC (e.g. loss of infeed from the wind farms) and the DC grid (e.g. converter loss) lead to deviation of frequency in the $\mathrm{AC}$ system depending on the governor droop characteristics [2]. Appropriate control of the MTDC grids to reduce such frequency deviations is the subject matter of this paper. One particular issue is how would an

Support from EPSRC, UK under grant EESC P11121 is acknowledged.

N.R. Chaudhuri and B. Chaudhuri are with Imperial College London, London, SW7 2BT, UK (e-mail: nilanjgec@gmail.com,b.chaudhuri@imperial.ac.uk).

R. Majumder is with SIEMENS Energy, Cary, NC 27511, USA (email:rajat.majumder@siemens.com).
MTDC grid react to loss/outage of one or more converters and the resulting power imbalance. Sharing the burden of such a loss/power mismatch has to be appropriate in order to minimize the impact on the neighboring AC system. Although, a few papers addressed the issues of VSC MTDC modeling [3], control strategies [4], [5], [6], protection [7] and power sharing using the concept of droop control [8]; hardly any except [9] has considered the problem of system frequency support through MTDC grids. In [9], a frequency support strategy based on local DC bus voltage - frequency droop is proposed. Interestingly, it resulted in different initial (i.e. steady-state) conditions in presence of frequency droop than without it. Moreover, no basis for selecting the controller parameters was established through modal analysis. Also in [9] a generic asymmetric bipole configuration was not considered which rules out studies with only one converter outage in a converter station.

In this work, frequency support for the surrounding $\mathrm{AC}$ systems interconnected through an asymmetric bipole MTDC grid with metallic return network is analyzed. The standard autonomous power sharing (droop) control [10] is modified by a frequency droop control loop to share the power mismatch in order to minimize the deviation in the AC system frequency. The effectiveness of the frequency support is established through nonlinear simulation of an MTDC grid with four converter stations connected to three isolated AC systems. Averaged model for the MTDC grid is developed with a view to do modal analysis of the combined AC-MTDC systems. Modal analysis is done to establish the sensitivity of the eigenvalues with respect to different droop coefficients, control parameters and change in operating condition involving converter outage. It is shown that, the frequency droop control for the converters in an MTDC grid is effective in reducing the deviation in frequency of the $\mathrm{AC}$ system following disturbances on both $\mathrm{AC}$ (loss of wind farm) and DC (converter outage) sides.

The main contributions of this paper are:

1) Propose new frequency droop control architecture for an asymmetric bipolar MTDC grid employing a common converter DC bus voltage.

2) Analyze the effect of varying the controller parameters through modal analysis and establish their boundary from stability point of view.

3) Evaluate the effectiveness of the proposed strategy following different disturbances including converter station outages. 


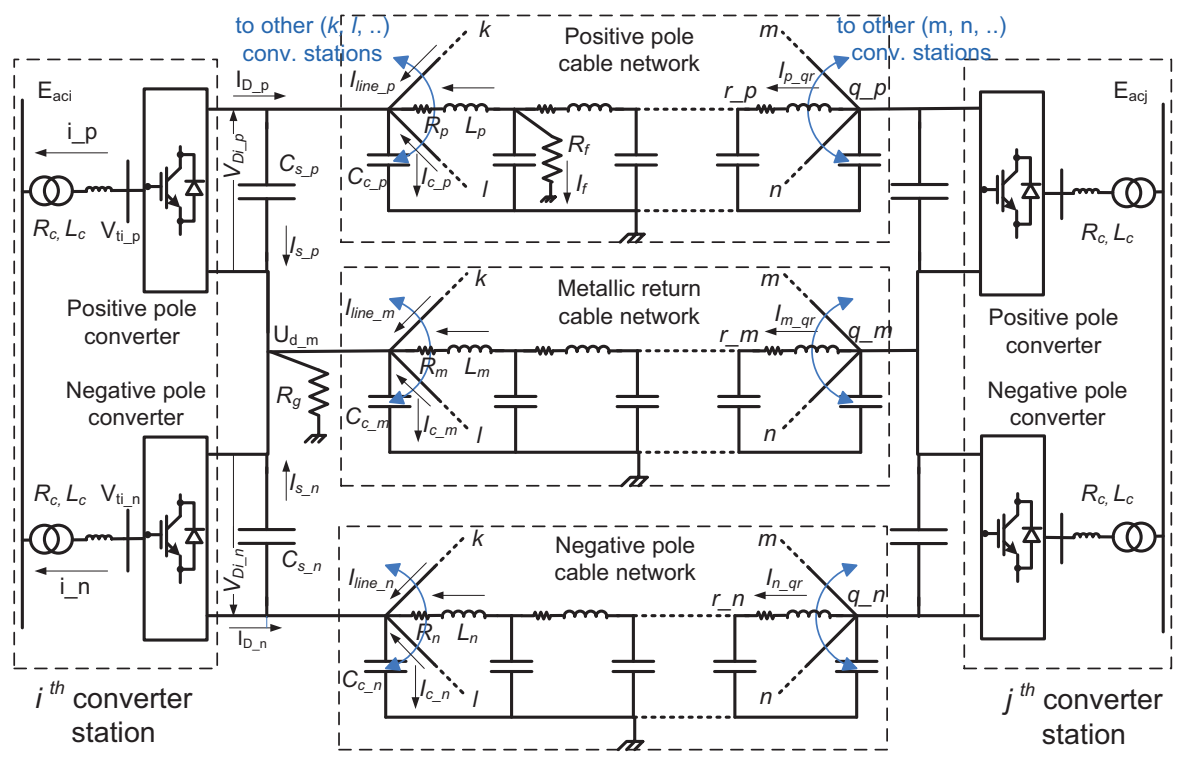

Fig. 1. DC cable network modeling with cascaded pi sections

\section{Modeling}

An asymmetric bipole MTDC grid with positive, negative and metallic return network was considered which is generic enough to handle different grounding mechanisms and 'unbalance on the DC side' as a result of outage of one or more poles and/or converters.

\section{A. Converter Modeling}

We have followed a similar approach as in [11] for modeling the converters which were represented by their averaged model [12] in Matlab/SIMULINK. This is elaborated very briefly here due to space restriction. Readers can refer to the extensively reported literature for further details.

The model of positive/negative pole of the $j^{t h}$ converter station shown in Fig. 1 was represented in a synchronously rotating reference frame $q^{\prime}-d^{\prime}$ (Fig. 2). The $d^{\prime}$-axis is locked with the voltage $E_{a c j}$ on the AC system side of the converters (see, Fig. 1) to ensure decoupled control of the active and reactive power [13]. In this work, the PLL dynamics was neglected. The assumption was that, the PLL can track changes in the system frequency accurately and adjust the speed of rotation of the reference frame accordingly. All notations in the modified reference frame are henceforth denoted with a prime.

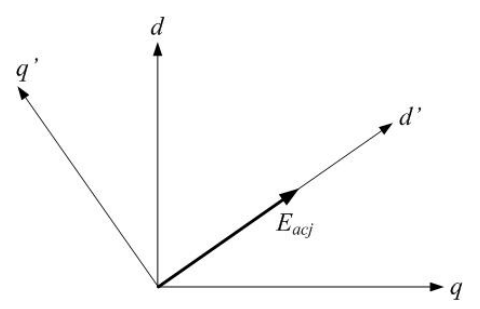

Fig. 2. Rotating $d-q$ reference frame for AC system modeling and modified $q^{\prime}-d^{\prime}$ reference frame for converter modeling and decoupled control
The AC system connected to the MTDC grid was modeled in $d-q$ reference frame as shown in Fig. 2 which needs to be transformed to $q^{\prime}-d^{\prime}$ frame and back while interfacing the MTDC grid variables with those of the AC system. The dynamical equations of the AC side of the converters in $q^{\prime}-d^{\prime}$ frame are not repeated here due to space restriction.

\section{B. DC Cable Network Modeling}

A cascaded pi section approximation of the distributed model of the cable as shown in Fig. 1 was used. The number of pi sections to be considered is a compromise between computational burden and accuracy. In [14] it was shown that 4 pi sections in the averaged model were adequate to match the impedance spectra up to the fundamental frequency. Obviously, the choice of number of pi-sections has to be made on a case to case basis.

Fig. 1 shows the interconnection between the $i^{t h}$ and the $j^{\text {th }}$ converter stations through the positive, negative and the metallic return cable. Each of the converter poles can be connected to the corresponding poles of any number of converter stations.

The mathematical model of the cable network can be described by the set of differential and algebraic equations (DAEs) shown in a block diagram in Fig. 3. The incidence matrix $C C I_{P}$ relates the vector of $R-L$ branch currents flowing from any general node $q \_p$ to $r \_p$ (i.e. $\bar{I}_{p_{-} q r}$ ) with the total node current injection vector $\bar{I}_{\text {line_p }}$ (see, Fig. 1) by the relation

$$
\bar{I}_{\text {line_p }}=C C I_{P} \times \bar{I}_{p_{-} q r}
$$

where, $C C I_{P}(i, j)=1(-1)$, if the $i^{t h}$ cable enters (leaves) the $j^{\text {th }}$ node and $C C I_{P}(i, j)=0$, otherwise. Note that $C C I_{P}=-C C U_{P}^{T}$. The branch $R_{f}$ and its current $I_{f}$ in Fig. 1 represents the fault resistance and the fault current for a DC cable fault.

The governing DAEs for the MTDC grid can be written from Figs 1 and 3 and are not repeated here since modeling 


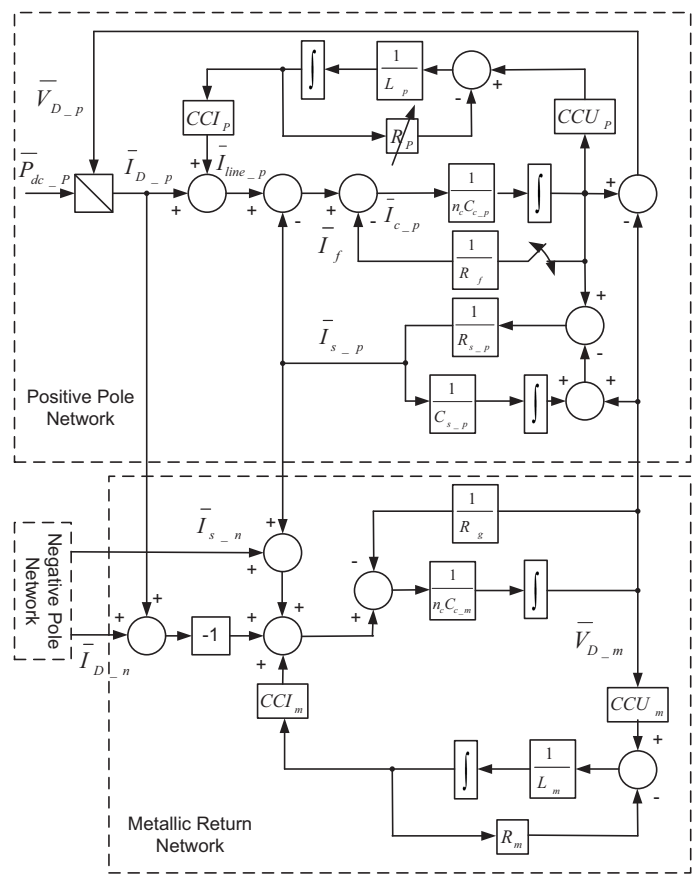

Fig. 3. Block diagram of the DC cable network dynamic model

is not the focus of this paper. The readers are referred to [14], [15] for further details. It should be noted that the converter outage was simulated by opening both the DC side and the AC side breakers at the respective converter pole. However, the DC cable connections to the switchyard of the affected converter were retained. Practical realization of VSC-based MTDC grids is based on the presumption of fault current limiting capability in VSC systems which is still not commercially feasible at high power levels. For the study reported in this paper it assumed that DC circuit breakers would be commercially available at high power levels at some point in future.

\section{Combined AC-MTDC Grid Modeling}

The model of the combined AC-MTDC grid is shown in Fig. 4. In the multi-machine $\mathrm{AC}$ system the generators, excitation systems, loads and other dynamic components were modeled by their respective differential and algebraic equations (DAEs). The AC network was represented algebraically by the bus admittance matrix $Y_{\text {bus }}$. A set of current balance equations including the current injection from the MTDC grid $\vec{I}_{M T D C}$ were solved to find the AC network bus voltages.

The voltages at the PCC of the MTDC grid $\left(\vec{E}_{a c}\right)$ were locked with the $d$-axis of the synchronously rotating $q^{\prime}-d^{\prime}$ reference frame (Fig. 2(b)). The converter AC side current was transformed into the $\mathrm{AC}$ system common $d-q$ reference to solve the current balance equations [14], [15].

\section{DROOP CONTROL}

For point-to-point VSC-HVDC links, the usual practice is to operate one of the converter stations in active power control mode to ensure the scheduled power exchange. The DC link voltage is maintained by the other station which consequently

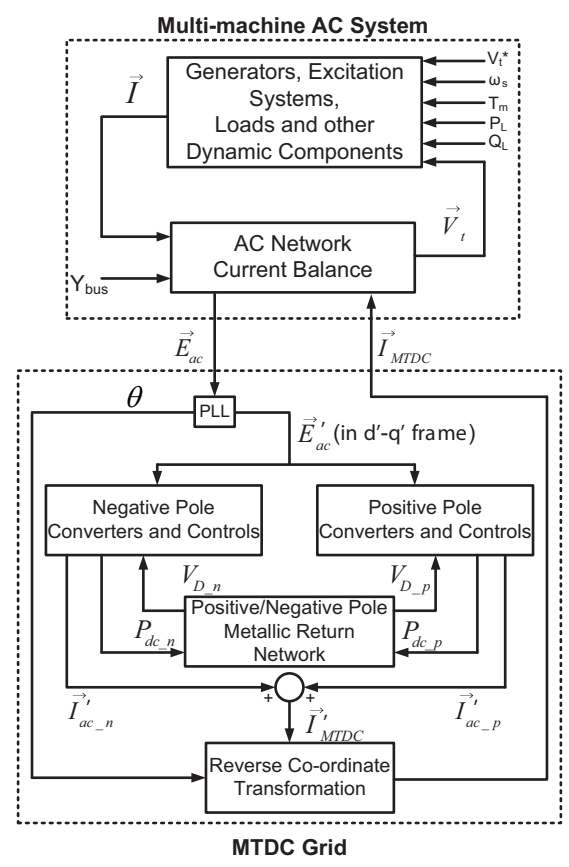

Fig. 4. Block diagram of combined AC-MTDC grid model

supplies the resistive losses in the DC link, thus acting as a slack/swing converter station.

Similarly, for an MTDC grid with more than two converter stations, one can be left to operate as a slack converter. The problem however, is that, following the outage of one or more converters, the slack converter would experience the entire burden of the change in power flow in the DC grid. The AC grid connected to the slack converter would also experience a sudden change in power import/export leading to frequency variation in the $\mathrm{AC}$ system depending on the size of the AC grid and the load flow scenario. For a converter station importing/exporting significant amount of power, this loss might lead to unacceptable variation in frequency. To avoid this, it is desirable that the converters share the resulting power imbalance in an appropriate proportion. An appropriate droop control scheme aimed at autonomous power sharing and frequency support can resolve these issues as described in the following sections.

\section{A. Autonomous Power Sharing}

To achieve autonomous power sharing, all the converter stations should operate in the DC link voltage control mode (or as swing converter) rather than trying to follow their respective active power references. However, set values of the DC link voltage references at all the converter stations could be conflicting unless they are modified properly depending on the reference and actual power and the DC link voltage.

The situation is similar to parallel operation of generators in an AC system where power-frequency droop is used in the governor control loop to avoid potential conflict in frequencies. Along the same lines, active power-DC link voltage droop can be used to modify the reference values of DC link voltages $V_{D j}^{2}$ at each converter station (under voltage control mode) as shown in Fig. 5. 


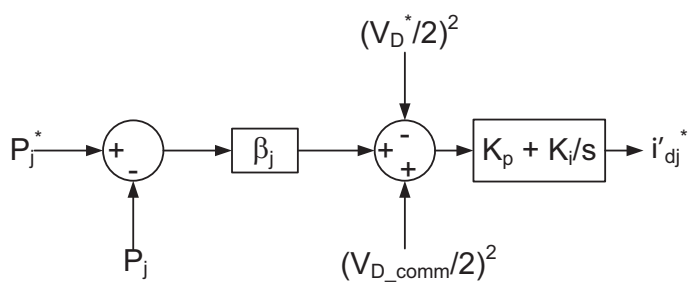

Fig. 5. Active power-DC link voltage droop for sharing of power imbalance in the $j^{\text {th }}$ converter station

Using the modified voltage reference, a PI controller derives the $d$-axis current reference $I_{d j}$ for the current control loop of the converters. Squared values of half of the the reference and measured DC link voltages $\left(\frac{V_{D j}{ }^{*}}{2}\right)^{2},\left(\frac{V_{D-c o m m}}{2}\right)^{2}$ are normally used for the voltage control loops [16]. Also local voltage feedback results in a non-unique power flow solution for the MTDC grid. Hence, a common voltage $V_{D-c o m m}$ (at any converter station) is communicated as feedback signal to the control loop of the remaining converter stations [10].

The values of the droop constants $\beta_{j}$ at different converter stations determine how a power imbalance is shared among the converters. Identical values of $\beta_{j}$ would result in equal sharing among the converters. For unequal $\beta_{j}$, the ones with higher values would have dominant contribution from active power $\left(P_{j}{ }^{*}\right)$ control loop. Smaller $\beta_{j}$ would ensure lesser deviation in DC link voltages, but it might adversely impact the stability as discussed later in Section V.

In an asymmetric bipole structure, the autonomous power sharing takes place only amongst the affected converter poles. For example, following the outage of a negative pole converter, the remaining negative pole converters will participate in power sharing, while the positive pole converters keep on operating independently at their pre-contingency operating condition [10].

\section{B. Frequency Support}

Autonomous power sharing ensures that none of the converters take the entire burden, which reduces the change in power import/export in the surrounding $\mathrm{AC}$ systems and the frequency deviation also get distributed amongst different $\mathrm{AC}$ grids connected to different converter stations. However, such a control strategy still results in inappropriate frequency deviations in different $\mathrm{AC}$ systems due to the following reasons:

- Following a disturbance in the AC side, e.g. step change in a generator torque reference, the frequency of that system changes due to governor action. The autonomous power sharing loop does not aid in reducing the frequency deviation, since there is no change in the net power exchange with the concerned $\mathrm{AC}$ grid.

- Change in the real power reference of a converter station results in appropriate distribution of the power deviation amongst different converter stations. Depending on how many stations are connected to each AC grid and the governor droop coefficients of different AC systems, this power sharing may result in larger frequency deviations in some $\mathrm{AC}$ systems.
- Following the outage of a converter in one pole, those in the unaffected pole do not participate in power sharing. Therefore, frequency deviation in certain $\mathrm{AC}$ systems might be large.

- Autonomous power sharing control alone can not appropriately share the frequency support (as an ancillary service) among AC systems interconnected through the MTDC grid.

In order to change the power sharing amongst different converter stations for the frequency support of the adjacent $\mathrm{AC}$ systems, the real power reference of the converter stations were modified with a supplementary frequency droop control as shown in Fig. 6 . The AC system frequency $f_{j}$ at the PCC of the $j^{t h}$ converter was used as a feedback signal to produce the frequency error, which in turn was multiplied by the frequency droop constant $\beta_{f j}$ to change the power reference.

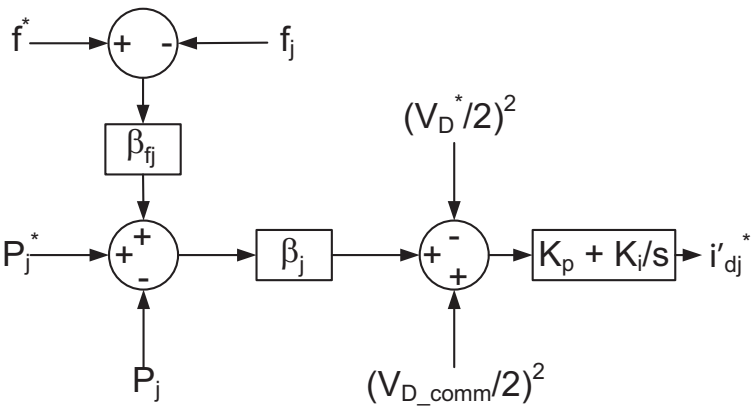

Fig. 6. Active power-DC link voltage droop for sharing of power imbalance in the $j^{t h}$ converter station and frequency support of the adjacent AC system

Here, $P_{j}^{*}$ is considered positive, if the station is acting as an inverter. Upon reduction (increase) in frequency, the real power injection into the $\mathrm{AC}$ system is commanded to increase (reduce) by changing the reference accordingly (Fig. 6). Note that, both the converter poles participate in the frequency support.

\section{STUdy Network}

Fig. 7 shows the study network consisting three asynchronous AC systems interconnected through the MTDC grid. One AC system has four interconnected generators $(G 1-G 4)$ and each of the two other AC grids consist of a single equivalent generator.

The generators $(G 1-G 6)$ were represented by their subtransient model, and were equipped with their governor loops. The active component of the loads were modeled as constant current and the reactive component as constant impedance. The details of the 4-machine AC system can be found in [2]. Other than the differential equations involving the converter transformers and phase reactors, the rest of the AC circuit (generator transformers, and transmission lines) was represented algebraically.

The MTDC grid consisting of four bipole converter stations were connected with $500 \mathrm{~km}$ cables as shown in Fig. 7. Converter stations \#2 and \#3 were considered to be acting as inverters while \#1 and \#4 as rectifiers under nominal condition. The DC link voltage of station \#1 was chosen as 


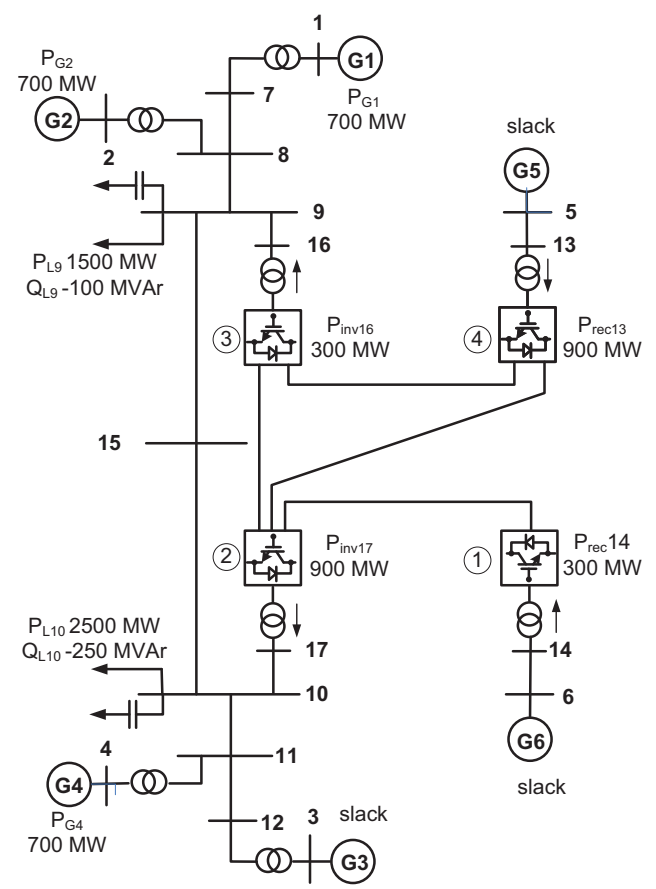

Fig. 7. Bipole MTDC grid (single line diagram) connected to a multi-machine AC system

the common reference for the droop scheme. The metallic return was grounded with a $0.5 \Omega$ resistance at the converter station \#1 while the other stations were left ungrounded. The converter stations were connected to the AC system through feeders of impedance $0.3+j 4.0 \Omega$ (the feeders connect buses $\# 5$ with \#13, \#6 with \#14, \#9 with \#16 and \#10 with \#17, see Fig. 7). The parameters of the MTDC grid can be found in [14], [15].

Under the nominal condition, each of $G 1, G 2$ and $G 4$ generates $700 \mathrm{MW}$ and the rest were left as slack. The tieline transfer through the AC line connecting buses \#9 and \#10 was around $200 \mathrm{MW}$ while all converter stations operated in droop control mode while maintaining unity power factor at the PCC as shown in Fig. 7.

\section{Sequential AC-DC Load Flow}

The load flow solution of the combined AC-MTDC systems was used to calculate the initial conditions of the states of the dynamic model. The AC-DC load flow was carried out in Matlab following a sequential approach as elaborated below:

1) First, the AC system was considered for the load flow, where $G 3, G 5$ and $G 6$ were assumed to be slack generators and the converter buses \#13, \#14, \#16 and $\# 17$ as load buses. Since the real power at these buses were not known before the load flow solution as the converter stations were working under droop control, they were initialized with their respective reference values. Assuming a load convention w.r.t the AC systems (i.e. $\mathrm{P}$ is positive (negative) if it is a load (generation)), the real power at the concerned buses were initialized as $900,300,-300$ and -900 MW respectively.
2) The voltage magnitudes and angles of buses \#5, \#6, \#9 and $\# 10$ (denoted in complex polar form as $V_{5}, V_{6}, V_{9}$ and $V_{10}$, respectively in Table I), calculated from the AC load flow in step 1, were used as initial guess for the DC load flow (in fact voltages at bus \#5 and \#6 were $1 \angle 0$ since they were slack buses).

3) The DC load flow solution in step 2 gave the modified real powers at buses \#13, \#14, \#16 and \#17 (denoted as $P_{13}, P_{14}, P_{16}$ and $P_{17}$, respectively in Table I). With these updated values, the AC load flow was run again.

4) Steps 1-3 were repeated until the maximum value of $\Delta P$ at the converter PCC buses (denoted as $\Delta P_{p c c-\max }$ in Table I) fell below a certain tolerance $\epsilon$ (assumed $10^{-10}$ in this work). Here, $\Delta P=P(k)-P(k-1)$ and $k$ is the number of iterations.

Three iterations of sequential AC-DC loadflow leading towards convergence are shown in Table I for the study system in Fig. 7. There is virtually no difference between the bus powers and voltages from iteration 2 to 3 as the maximum value of the power mismatch at the converter PCC buses $\left(\Delta P_{p c c-\max }\right)$ was already at a low value of $1.57 \mathrm{E}-06$.

TABLE I

ITERATIONS LEADING TO CONVERGENCE OF SEQUENTIAL AC-DC LOADFLOW

\begin{tabular}{|l||c|c|c|}
\hline AC-DC loadflow iter. & 1 & 2 & 3 \\
\hline \hline$P_{13}$ & 9.0 & 9.0341 & 9.0341 \\
\hline$P_{14}$ & 3.0 & 3.0341 & 3.0341 \\
\hline$P_{16}$ & -3.0 & -2.9659 & -2.9659 \\
\hline$P_{17}$ & -9.0 & -8.9659 & -8.9659 \\
\hline$V_{5}$ & $1 \angle 0$ & $1 \angle 0$ & $1 \angle 0$ \\
\hline$V_{6}$ & $1 \angle 0$ & $1 \angle 0$ & $1 \angle 0$ \\
\hline$V_{9}$ & $0.98 \angle-15.72$ & $0.98 \angle-16.17$ & $0.98 \angle-16.17$ \\
\hline$V_{10}$ & $0.99 \angle-26.66$ & $0.99 \angle-26.89$ & $0.99 \angle-26.89$ \\
\hline$\Delta P_{\text {pcc-max }}$ on & 0.0341 & $1.57 \mathrm{E}-06$ & $7.31 \mathrm{E}-11$ \\
\hline No of AC loadflow iter. & 15 & 9 & 5 \\
\hline No of DC loadflow iter. & 4 & 4 & 4 \\
\hline
\end{tabular}

\section{Modal Analysis}

The MTDC grid model described by differential-algebraic equations (DAEs) was linearized around a nominal condition. Participation factor analysis was done to figure out the nature and the root cause of the dynamic response of the MTDC grid. It was found that the poles in the far left of the splane has more participation from the states of the converter transformer and phase reactor (i.e. AC side states). As we move towards the right, the participation from the DC network, machine and droop controller states increase. To establish a stability zone for the droop controller gains $\beta$ (pu $k V^{2} / \mathrm{MW}$ ), $\beta_{f}(\mathrm{MW} / \mathrm{Hz}), K_{p}$ (pu MW/kV $V^{3}$ ) and $K_{i}$ (pu MW-s/kV $V^{3}$ ) the sensitivity of the eigenvalue movement w.r.t these parameters were investigated (for the pu calculations, the base quantities used were: $\mathrm{MVA}=608, \mathrm{kV}=120.09$ ).

For $\beta=0.1$ and $\beta_{f}=100$, the value of $K_{p}$ was increased from 0.2 to 1.0. The integral coefficient $K_{i}$ was kept 10 times that of $K_{p}$. It can be seen from the root locus plot of Fig. 8 that there is a mixed movement of the poles - some towards further left and some towards right. One of the DC network modes have moved in the right half of the s-plane, making the 
system unstable. Therefore, it can be concluded that increasing the PI controller gains can reduce system stability.
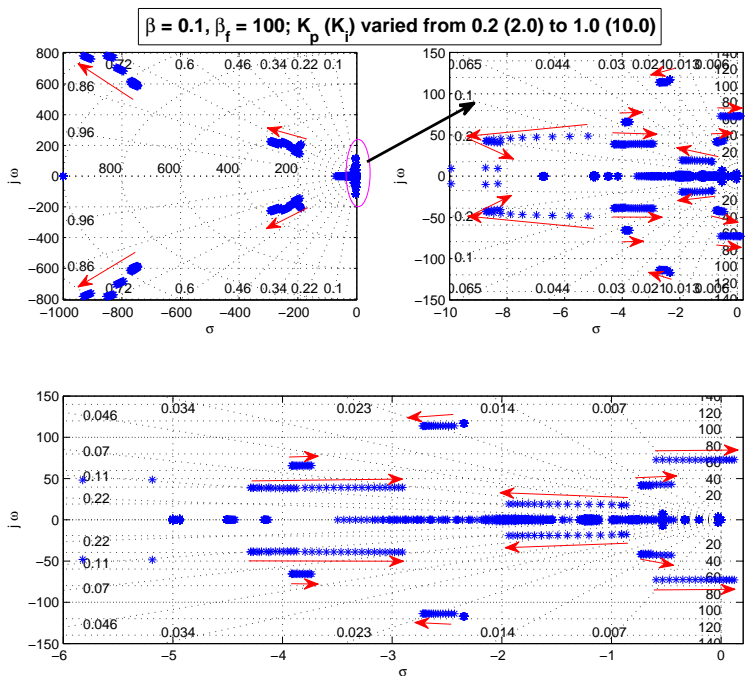

Fig. 8. Root locus of system poles with increase of $K_{p}$ from 0.2 to 1.0, keeping $\beta$ and $\beta_{f}$ constant at 0.1 and 100 respectively. The value of $K_{i}$ is kept 10 times that of $K_{p}$.

To find out the implication of variation in $\beta$ on the system stability the values of $K_{p}, K_{i}$ and $\beta_{f}$ were fixed at $1.0,10.0$ and 100.0 respectively. When $\beta$ is reduced from 0.5 to 0.1 the system becomes unstable as shown in Fig. 9.
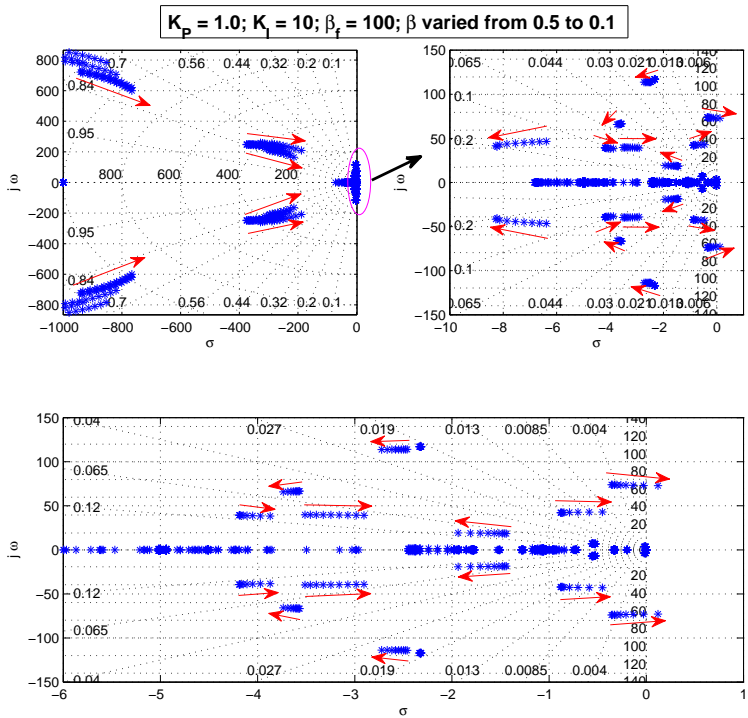

Fig. 9. Root locus of system poles with reduction of $\beta$ from 0.5 to 0.1 , keeping $K_{p}, K_{i}$ and $\beta_{f}$ constant at $0.1,10.0$ and 100.0 respectively.

It is clear from this exercise that increase in $\beta$ can increase the system stability as opposed to $K_{p}$ and $K_{i}$. Also worth noting is the movement of the AC network poles in the same direction of the critical poles of the system towards the right half of the s-plane.

These studies provide invaluable insight into the root-cause analysis of the system dynamic behavior and stability. We also found that $\beta_{f}$ has a low sensitivity towards the movement of the system eigenvalues. After establishing the stability zone at nominal condition, the following values were considered for the droop controller $\beta=0.1, \beta_{f}=100.0, K_{p}=0.5$ and $K_{i}=5.0$. However, it was found that following the outage of one pole of converter station \#3 the system becomes unstable as shown in Fig. 10.
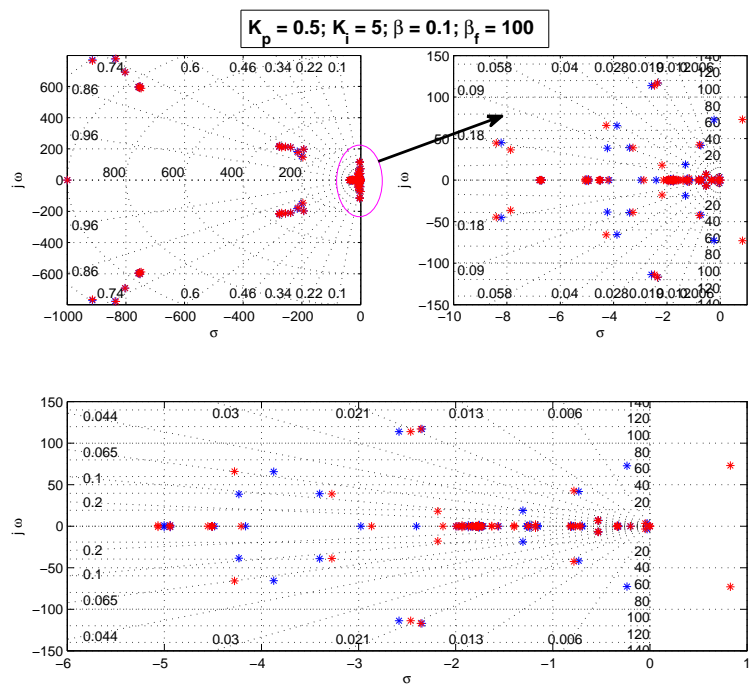

Fig. 10. System pole locations following the outage of the negative pole of converter station \#3. The pole map in red presents the converter outage condition while its blue counterpart show that of nominal condition.

This highlights the importance of considering different contingency scenarios during the design process. Also, it was found that the operating condition alters the sensitivity of the eigen-value movements. Finally, the following values were chosen for the droop control: $\beta=0.1, \beta_{f}=100.0, K_{p}=0.1$ and $K_{i}=1.0$.

\section{Simulations AND Discussion}

Non-linear time-domain simulations were done to demonstrate frequency support provision among AC systems interconnected through an MTDC grid. To simulate frequency events, disturbances were created on both AC and DC sides. Disturbances on the AC side include step change in mechanical torque input of generators as well as changes in load. On the DC side, change in power reference command at certain converter stations and outage of one pole (either positive or negative) is considered.

\section{A. AC Side Disturbances}

An sudden loss of infeed to the MTDC grid is one of the major concerns of the system operators as this will impact other AC grids connected to it. To emulate such a disturbance we have considered step change in the mechanical torque input of the generators.

1) Step Reduction in Mechanical Torque Input of G1:

Fig. 11 shows the variation in the system frequency due to a step reduction in the torque reference of $\mathrm{G} 1$.

The frequency of the interconnected 4-machine AC system reduces below $59.5 \mathrm{~Hz}$. During dynamic condition the 

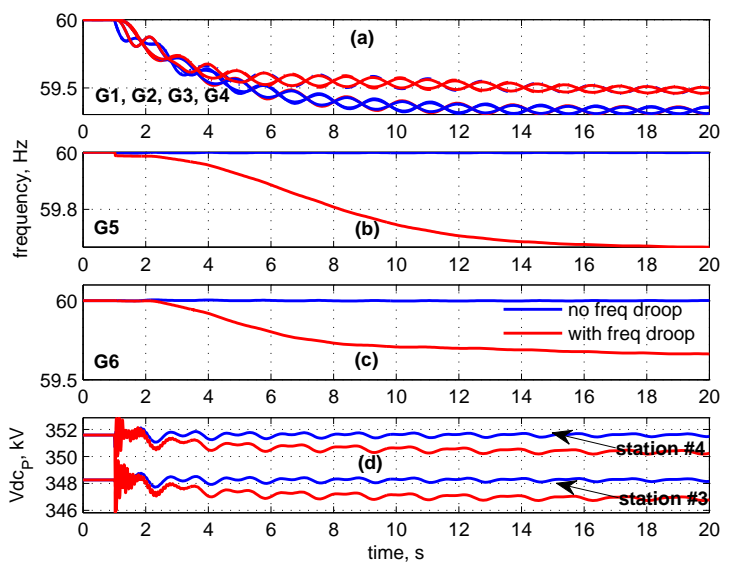

Fig. 11. AC system frequency and MTDC DC link voltage variation following a step reduction of torque reference of G1.

generator frequencies are different from each other - varying according to the modeshapes of the generator speeds. The frequency of the two isolated power systems corresponding to G5 and G6 remain unchanged as they are decoupled from the 4-machine AC system by the MTDC network.

Without frequency droop, the real power at the point of common coupling $\left(P_{P C C}\right)$ of all converter stations oscillate about the pre-disturbance average value as shown in Fig. 12.
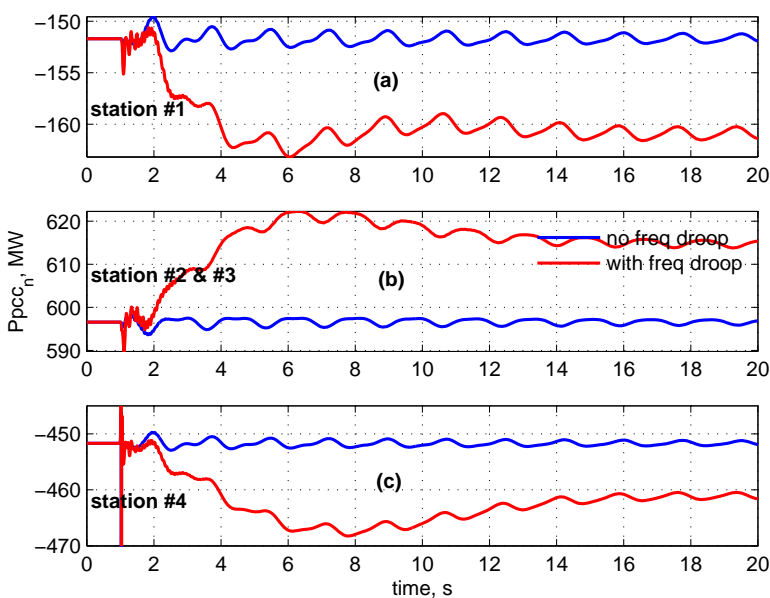

Fig. 12. Dynamic behavior of real power at PCC of the negative pole MTDC converters following a step reduction of torque reference of G1.

Only negative pole real powers are shown due to symmetry - i.e. identical behavior is observed in the positive pole converters. In presence of frequency droop the total real power injection into the 4-machine AC system increases, see Fig. 12(b). This compensates the reduction in the torque of G1 and restricts the frequency from going below $59.5 \mathrm{~Hz}$ as shown in Fig. 11(a).

Closer look reveals that this frequency support comes at the expense of increase in real power drawn by the two other converter stations \#1 and \#4 from the two isolated power systems. This results in reduction of frequencies of those power systems, as shown in Figs 11(b) and (c).

2) Step Reduction in Mechanical Torque Input of G5: As shown in Fig. 13, the effect of frequency support through the MTDC grid is more pronounced for a step reduction in power of G5. In absence of frequency droop, the frequency of this system goes well below $58.0 \mathrm{~Hz}$ while the frequency droop results in only $1 \mathrm{~Hz}$ variation at the expense of minimal (less that $0.5 \mathrm{~Hz}$ ) change in frequency of the two other AC systems. Note the resulting change in the DC link voltages for both the disturbances which are acceptable (within $\pm 5 \%$ ).

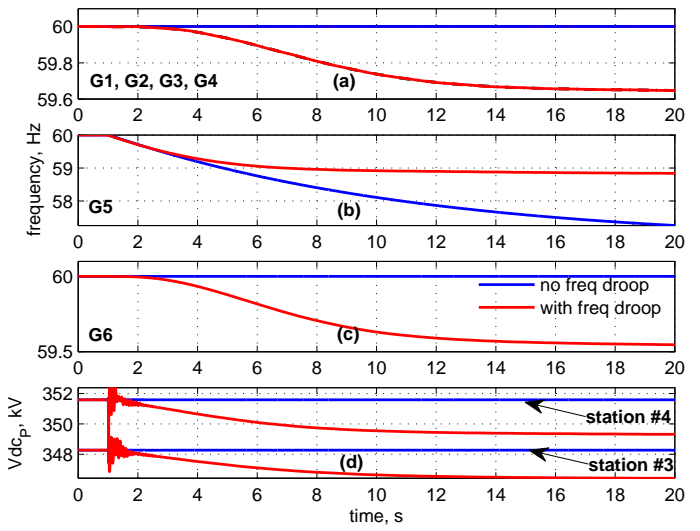

Fig. 13. AC system frequency and MTDC DC link voltage variation following a step reduction of torque reference of G5.

It is interesting to note that a disturbance in the interconnected 4-machine 2-area AC system resulted in observable inter-area mode in the frequency of the generators G1-G4 (Fig. 11(a)). On the other hand a similar disturbance at the isolated AC system G5 did not trigger such oscillations as shown in Fig. 13(a).

3) Step Increase in Load at Bus 10: The variation of frequency in the AC networks surrounding the MTDC grid is shown in Fig. 14 in response to a 1\% step increase in load connected at bus 10 .
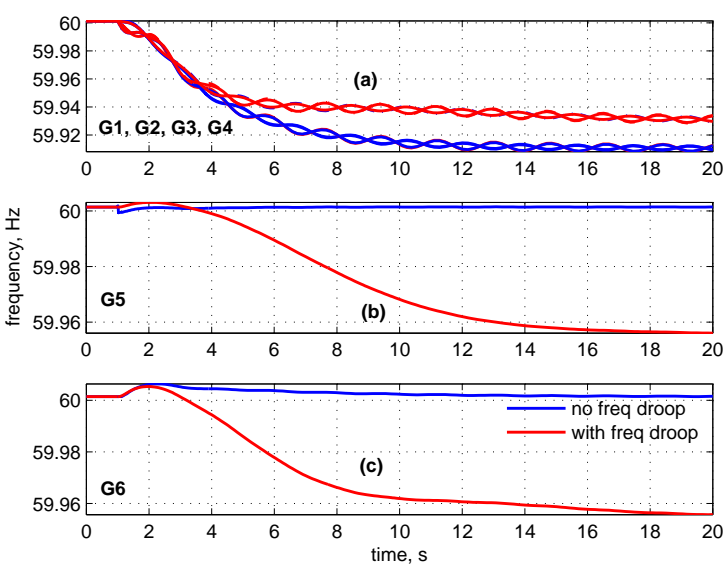

Fig. 14. AC system frequency variation following a step increase in load at bus 10 .

As expected the frequency variation in the 4-machine in- 
terconnected AC system is more when there is no frequency droop. However, with droop control frequency support is provided from the remote AC systems (G5, G6) through the MTDC grid which results in less frequency variation.

The variation of real power at the PCC of the negative pole MTDC converter is shown in Fig. 15. From Fig. 15(b) it can be seen that in presence of frequency droop the total real power injection into the 4-machine AC system increases to cater for the increase in load. This excess power for the 4-machine system is provided by the two isolated power systems through converter stations \#1 and \#4 as seen in Fig. 12(a),(c). This results in reduction of frequencies (but within allowable limits) of those power systems, as shown in Figs 14(b) and (c).
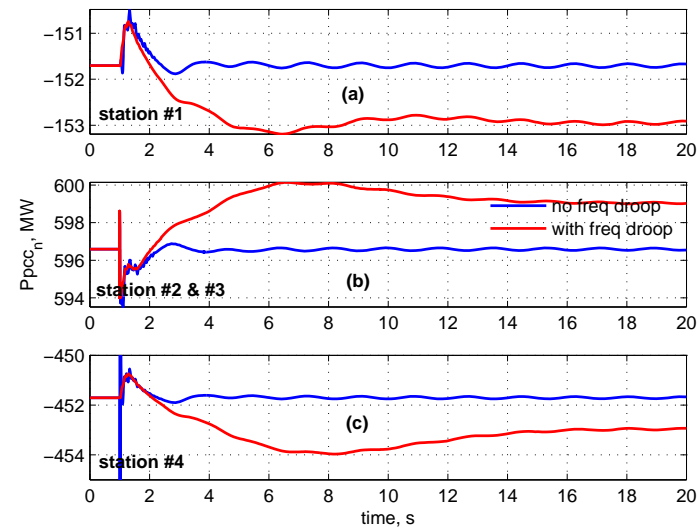

Fig. 15. Dynamic behavior of real power at PCC of the negative pole MTDC converters following a step increase in load at bus 10 .

\section{B. Converter Station Disturbances}

1) Step Reduction in Pref of Station \#3: After studying frequency deviation due to AC side disturbances, the effect of converter side disturbance is investigated. The variation in $\mathrm{AC}$ system frequency can be seen from Fig. 16 following a step reduction of the real power reference of each pole of converter station \#3 by $50 \%$ (i.e. from $150 \mathrm{MW}$ to $75 \mathrm{MW}$ ).
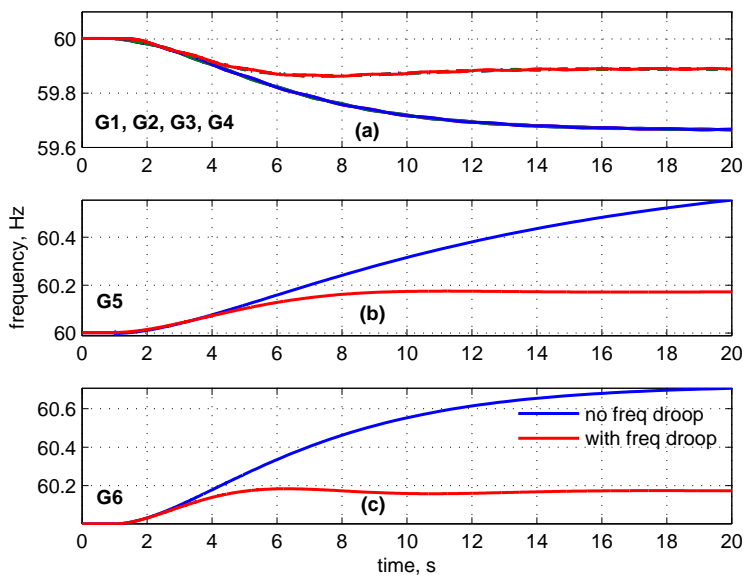

Fig. 16. AC system frequency variation following a step reduction of real power reference of converter station \#3.
As expected, without frequency droop, each converter pole of the four stations change their power by about $19 \mathrm{MW}$, see Fig. 17. It is shown in Fig. 17(b) that the net reduction in power injection from each pole of stations \#2 and \#3 is around 38 MW.
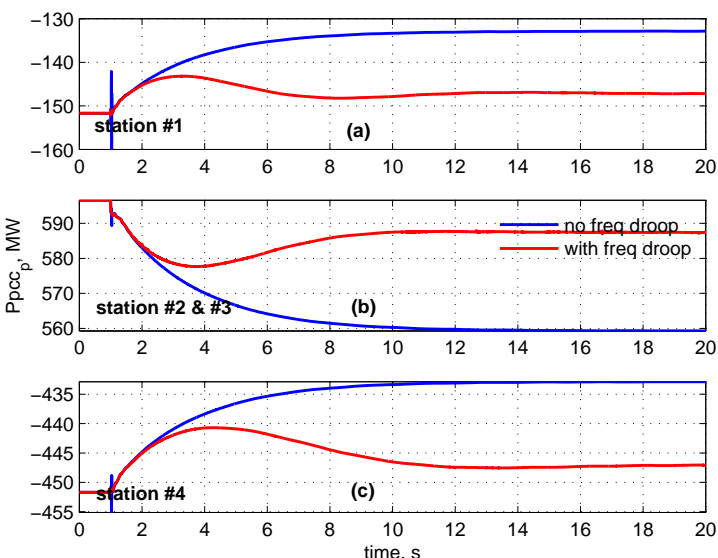

Fig. 17. Dynamic behavior of real power at PCC of the positive pole MTDC converters following a step reduction of real power reference of converter station \#3.

These changes have resulted in a drop in frequency of G1G4 by about $0.3 \mathrm{~Hz}$ and increase in frequency of G5 and G6 by about $0.6 \mathrm{~Hz}$ (Fig. 16). The cause of increase/decrease of frequency of the respective systems can be explained by the corresponding decrease/increse of system loads in terms of real power exchange at the PCC of the converter stations. On the other hand, the introduction of frequency droop reduces the change in converter powers at all stations, see Fig. 17. As observed from Fig. 16, this reduces the frequency variation in all the $\mathrm{AC}$ systems.

2) Outage of Negative Pole of Station \#3: The outage of the negative pole of converter station \#3 results in unbalanced operation in the DC side which is clear from the non-zero metallic return current shown in Fig. 18(d).

With only $P-V_{d c}$ droop, as the station \#3 negative pole power becomes zero (Fig. 19(c)), the rest of the negative poles share the lost power (i.e. $150 \mathrm{MW}$ ) equally amongst themselves resulting in $50 \mathrm{MW}$ deviation (shown in Fig. 19) for each.

Note that the aggregate $P_{p c c}$ of stations \#2 and \#3 negative poles are separately shown in Fig. 19(b) which changes by about $100 \mathrm{MW}$. It is worth noting that the positive pole control operates independently and the converter powers do not change from the pre-disturbance values in absence of frequency droop, see Fig. 20.

Similar to the previous case, the changes in the converter real powers resulted in deviation of $\mathrm{AC}$ system frequencies as shown in Fig. 18. Frequency of the 4-machine AC system reduces to $59.6 \mathrm{~Hz}$ while that of G5 and G6 increases to $60.8 \mathrm{~Hz}$ and $60.9 \mathrm{~Hz}$ respectively. It is interesting to observe the converter real power response with frequency droop for the positive pole (Fig. 20) and the negative pole (Fig. 19) respectively. Station \#1 reduces the net deviation in power 

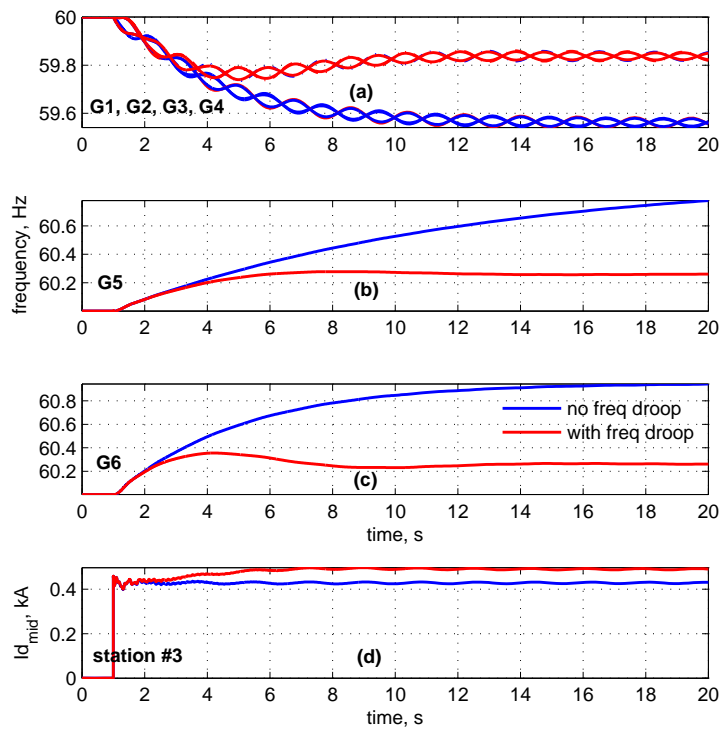

Fig. 18. AC system frequency and metallic return current variation following the outage of negative pole converter of station \#3.
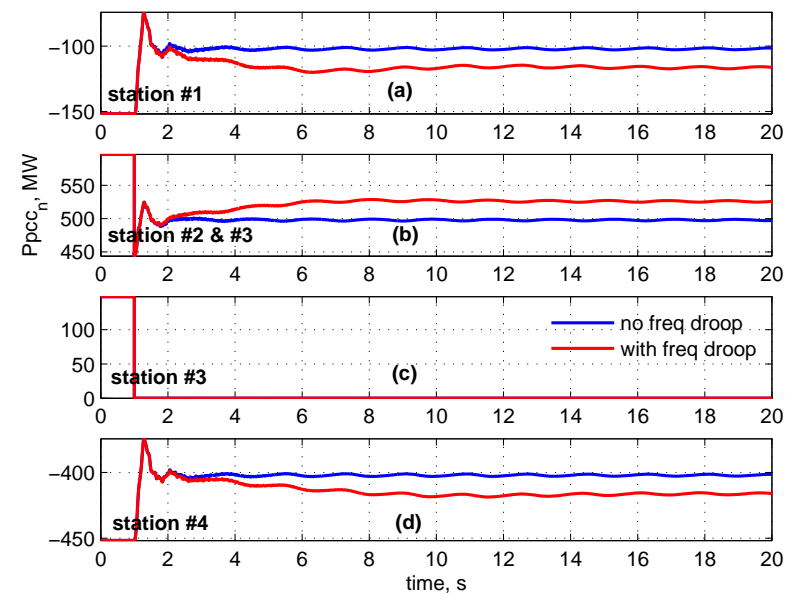

Fig. 19. Dynamic behavior of real power at PCC of the negative pole MTDC converters following the outage of negative pole converter of station \#3.

output from $50 \mathrm{MW}$ to around $15 \mathrm{MW}$. To be more specific, under post-disturbance condition $P_{p c c p}$ is approximately -170 MW and $P_{p c c n}$ is about -115 MW totalling about $-285 \mathrm{MW}$ - which is a $15 \mathrm{MW}$ deviation from the pre-disturbance -300 MW output. This trend is clearly visible for the other converter stations as well.

As a result the individual AC systems experience reduced change in system loads. Therefore, the frequency deviation of the 4-machine $\mathrm{AC}$ system is reduced to $0.2 \mathrm{~Hz}$ while that of G5 and G6 are less than $0.3 \mathrm{~Hz}$, see Fig. 18 .

\section{CONCLUSION}

Frequency support amongst AC systems interconnected through a multi-terminal DC (MTDC) grid has been demonstrated. The autonomous power sharing control has been modified with a frequency droop control loop to minimize

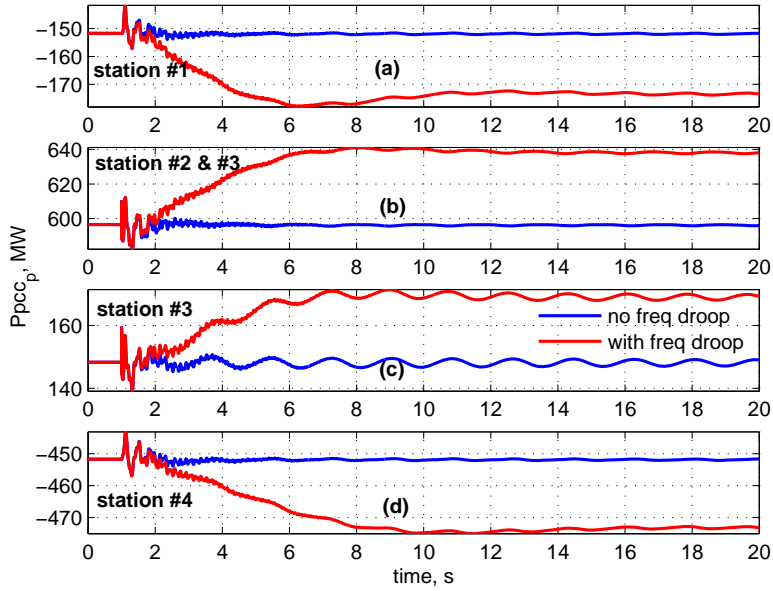

Fig. 20. Dynamic behavior of real power at PCC of the positive pole MTDC converters following the outage of negative pole converter of station \#3.

the deviation from nominal $\mathrm{AC}$ system frequency and share the burden of frequency support among the converter stations of the MTDC grid. An averaged model of the MTDC grids is developed to carry out modal analysis of combined multimachine AC-MTDC systems. The sensitivity of the system eigen-values to changes in control parameters (e.g. droop coefficients) has been established through modal analysis. It is shown that appropriate droop control loop for the MTDC grid converters could be effective in reducing the deviation from nominal AC system frequency.

\section{REFERENCES}

[1] J. Blau, "Europe plans a north sea grid," IEEE Spectrum, vol. 47, no. 3, pp. 12-13, 2010.

[2] P. Kundur, Power system stability and control, ser. The EPRI power system engineering series. New York; London: McGraw-Hill, 1994.

[3] C. Zheng, X. Zhou, and L. Ruomei, "Dynamic modeling and transient simulation for VSC based HVDC in multi-machine system," in International Conference on Power System Technology, 2006. PowerCon 2006, 2006, pp. 1-7.

[4] C. Hairong, W. Chao, Z. Fan, and P. Wulue, "Control strategy research of VSC based multiterminal HVDC system," in IEEE PES Power Systems Conference and Exposition, 2006. PSCE '06. 2006.

[5] X. Lie, Y. Liangzhong, and M. Bazargan, "Dc grid management of a multi-terminal hvdc transmission system for large offshore wind farms," in International Conference on Sustainable Power Generation and Supply, 2009. SUPERGEN '09, 2009, pp. 1-7.

[6] L. Fei, M. Zhiwen, Y. Xiaojie, and Z. Trillion, "The grid connected converter control of multi-terminal DC system for wind farms," in Proceedings of the Eighth International Conference on Electrical Machines and Systems, 2005. ICEMS 2005, vol. 2, 2005, pp. 1021-1023 Vol. 2.

[7] T. Lianxiang and O. Boon-Teck, "Locating and isolating DC faults in multi-terminal DC systems," IEEE Transactions on Power Delivery, vol. 22, no. 3, pp. 1877-1884, 2007.

[8] X. Lie, B. W. Williams, and Y. Liangzhong, "Multi-terminal dc transmission systems for connecting large offshore wind farms," in IEEE Power and Energy Society General Meeting - Conversion and Delivery of Electrical Energy in the 21st Century, 2008, 2008, pp. 1-7.

[9] T. M. Haileselassie and K. Uhlen, "Primary frequency control of remote grids connected by multi-terminal HVDC," in IEEE Power and Energy Society General Meeting, 2010.

[10] N. R. Chaudhuri, R. Majumder, and B. Chaudhuri, "Adaptive droop control for effective power sharing in multi-terminal dc (MTDC) grids," to appear in IEEE Transactions on Power Systems, 2012.

[11] A. Yazdani and R. Iravani, Voltage-sourced converters in power systems : modeling, control, and applications. Oxford: Wiley, 2010. 
[12] S. R. Sanders, J. M. Noworolski, X. Z. Liu, and G. C. Verghese, "Generalized averaging method for power conversion circuits," IEEE Transactions on Power Electronics, vol. 6, no. 2, pp. 251-259, 1991.

[13] C. Schauder and H. Mehta, "Vector analysis and control of advanced static var compensators," IEE Proceedings on Generation, Transmission and Distribution, vol. 140, no. 4, pp. 299-306, 1993.

[14] N. R. Chaudhuri, R. Majumder, B. Chaudhuri, and J. Pan, "Stability analysis of VSC MTDC grids connected to multi-machine AC systems," IEEE Transactions on Power Delivery, vol. 26, no. 4, pp. 2774-2784, 2011.

[15] N. R. Chaudhuri, R. Majumder, B. Chaudhuri, J. Pan, and R. Nuqui, "Modeling and stability analysis of MTDC grids for offshore wind farms: A case study on the north sea benchmark system," in proceedings of IEEE PES General Meeting, 2011.

[16] Z. Lidong, L. Harnefors, and H. P. Nee, "Power-synchronization control of grid-connected voltage-source converters," IEEE Transactions on Power Systems, vol. 25, no. 2, pp. 809-820, 2010.

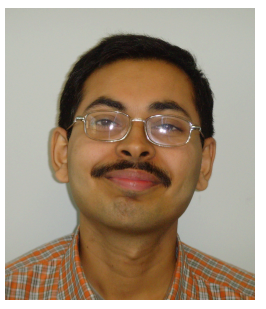

Nilanjan Ray Chaudhuri (S'08-M'09) received his Ph.D. in electrical power systems from Imperial College London in 2011. His research interests include power system dynamics and control, application of power electronics in power systems, online system identification, FACTS, HVDC and renewable energy systems.

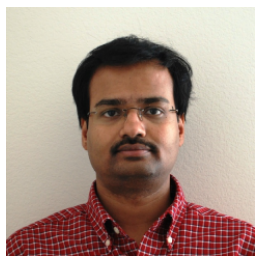

Rajat Majumder (S'03-M'06) received the Ph.D. degree in electrical power systems from Imperial College London, London, U.K., in January 2006. He is presently working for SIEMENS Energy, USA. He was with $\mathrm{ABB}$ Corporate Research in Raleigh, NC, USA and Vasteras, Sweden. He has also worked as a Lecturer in Power and Energy Systems at the University of Queensland, Brisbane, Australia, between 2006-2007. His research interest is power systems dynamics and control.

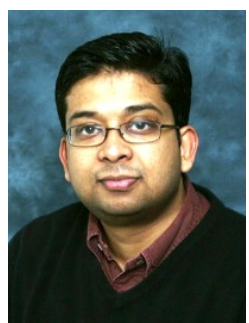

Balarko Chaudhuri (S'02-M'06-SM'11) received his $\mathrm{PhD}$ in electrical power systems from Imperial College London in 2005. He worked with General Electric Global Research for a year before coming back to Imperial College as a post-doctoral research associate in 2006 . He is presently a lecturer in the department of Electrical and Electronic Engineering at Imperial College London. His research interests are in the areas of power system dynamics and stability, FACTS/HVDC, robust control and renewable energy. 